\begin{tabular}{|l|l|}
\hline $\begin{array}{l}\text { Postprint } \\
\text { Version }\end{array}$ & 1.0 \\
\hline Journal website & http://onlinelibrary.wiley.com/doi/10.1111/jep.12096/abstract \\
\hline Pubmed link & $\underline{\text { http://www.ncbi.nlm.nih.gov/pubmed/24304535 }}$ \\
\hline DOI & $10.1111 /$ jep.12096 \\
\hline
\end{tabular}

This is a NIVEL certified Post Print, more info at http://www.nivel.eu

\title{
Surgical residents' perceptions of patient safety climate in Dutch teaching hospitals
}

\author{
KARTINIE MARTOWIRONO MSC JUNIOR RESEARCHER ${ }^{1, *}$, CORDULA WAGNER MA PHD \\ PROFESSOR OF PATIENT SAFETY ${ }^{2,3}$ AND A. BART BIJNEN MD PHD PROFESSOR OF \\ SURGERY ${ }^{1,4}$ \\ ${ }^{1}$ Foreest Medical School, Medical Center Alkmaar, Alkmaar, The Netherlands \\ ${ }^{2}$ EMGO Institute for Health and Care Research (EMGO+), VU University Medical Center, \\ Amsterdam, The Netherlands \\ ${ }^{3}$ NIVEL, The Netherlands Institute for Health Services Research, Utrecht, The Netherlands \\ ${ }^{4}$ VU University Medical Center, Institute for Education and Training, Amsterdam, The , \\ Netherlands
}

\begin{abstract}
Rationale, aims and objectives : Explicit attention to patient safety during surgical training is needed to improve patient safety. A positive safety climate is associated with greater patient safety and is a requisite for safety teaching at the workplace. The Safety Climate Survey (SCS) measures perceptions of safety climate. This study aims to take a first step in validating the SCS for use among surgical residents in the Netherlands and to highlight opportunities for safety climate improvement through changes in surgical training in the Netherlands. It therefore assesses (1) if the SCS can be used to assess surgical residents' perceptions of the safety climate in Dutch teaching hospitals; and (2) how, according to SCS results, these residents perceive the safety climate in Dutch teaching hospitals.

Methods : In a cross-sectional study conducted in February 2011, a Dutch translation of the SCS was administered to all general surgical residents in the Netherlands. Face validity and internal consistency were assessed, as were overall mean, means per item and significant differences in means between different groups of respondents.

Results : In total, 306 of 390 (78\%) residents completed the questionnaire. The SCS showed good face validity and internal consistency (Cronbach's alpha $=0.87$ ). Residents reported an overall mean of 3.95 (standard deviation 0.51 ) out of a maximum score of 5.00 , and $52 \%$ reported an overall mean of 4.00 or higher. Women and residents working in university hospitals gave
\end{abstract}


Martowirono, K., Wagner, C., Bijnen, A.B. Surgical residents' perceptions of patient safety climate in Dutch teaching hospitals. Journal of Evaluation in Clinical Practice: 2014, 20(2), 121-

significantly lower scores. Significant differences were also found among hospitals and among regions. Majority of the items scored less than 4.00.

Conclusions: The SCS is potentially useful to measure surgical residents' perceptions of the patient safety climate in Dutch teaching hospitals. There is considerable room for improvement of the patient safety climate. Surgical training should include better feedback, formal patient safety teaching sessions at the workplace and specific attention to patient safety during the introduction in a new hospital, and supervisors should encourage surgical residents to report any patient safety concern they may have.

\section{INTRODUCTION}

\section{Patient safety education during surgical training is needed to improve patient safety} Adverse events in health care cause considerable morbidity, mortality and costs [1, $2]$. A large proportion of in-hospital adverse events is surgery-related [2, 3]. Several efforts have been made to reduce the rate of adverse events in surgery. Among them were briefing tools and checklists, which have proven to improve quality and save costs and are nowadays used throughout the world [4-8]. Individual safety competencies have been formulated by the Canadian Patient Safety Institute, and team trainings such as TeamSTEPPS have been developed $[9,10]$. They aim to further reduce human error by enhancing safety consciousness of health care workers and by improving cooperation among individuals. Considerable investments in patient safety education are required to attain sustainable improvements in patient safety $[11,12]$.

In the Netherlands, patient safety courses are progressively integrated in postgraduate medical training. However, research showed that the long-term effect of a standalone patient safety course is limited. To achieve long-lasting effects, it is also recommended to integrate patient safety education in training at the workplace [13$16]$.

\section{Safety climate is one of the factors that influence patient safety and is essential for effective patient safety education at the workplace}

Several factors influence patient safety: institutional context, organizational and management factors, work environment, team factors, individual (staff) factors, task factors and patient characteristics $[17,18]$. One important component of organizational and management factors is safety culture. Safety culture is commonly defined as 'the product of individual values, group values, attitudes, perceptions, competencies and patterns of behaviour that determine the commitment to, and the style and proficiency of an organization's safety management' [19, 20]. Safety climate can be defined as the measurable components of safety culture [20,21]. Several studies report that a positive safety climate is associated with greater patient safety [22-25]. For teaching safety at the workplace, a positive safety climate is an essential requisite.

\section{The Safety Climate Survey measures perceptions of safety climate}

One of many surveys that measure safety climate in health care is the University of Texas Safety Climate Survey (SCS) [20,26-30]. It was developed by Sexton and colleagues, who extracted seven items related to safety climate from the larger Safety 
Attitudes Questionnaire. They added 14 items that had been linked to safety and performance outcomes in prior aviation research or were identified through discussions with hospital executives, quality experts and other end-users. This resulted in a 21-item instrument with sound psychometric properties [26, 29-31]. No subscales have been reported in literature. Compared with other questionnaires that measure safety climate, the SCS has less items, which makes it easier to administer and hence could raise the chance of a high response rate [20,27, 28]. The instrument was endorsed by the Institute for Healthcare Improvement. It has been used before, for instance among staff in a multi-centre intensive care unit (ICU) study in Canada, but no data of specific administration to surgical residents exist [30]. Furthermore, the questionnaire has not yet been validated for use in the Netherlands.

\section{Aims and objectives}

The Association of Surgeons in the Netherlands aims to optimize surgical training in the Netherlands with respect to patient safety. This study, which is funded by the association, takes a first step into evaluating the safety climate among surgical residencies and highlights opportunities for safety climate improvement through changes in surgical training in the Netherlands. It therefore examines (1) if the SCS is a potentially useful instrument, determined by the assessment of face validity and internal consistency, to assess surgical residents' perceptions of the safety climate in Dutch teaching hospitals; and (2) how, according to SCS results, these residents perceive the safety climate in Dutch teaching hospitals.

\section{METHODS}

\section{Research ethics}

Participation in this study was voluntary. Data were collected in such a way that they cannot be traced back to an individual.

\section{Data collection}

\section{Setting}

This cross-sectional study was conducted in the Netherlands in February 2011. In the context of this study, the term 'surgical resident' refers to a physician who finished medical school and who is in training to become a surgeon. These surgical residents do a part of their training in one of the eight university hospitals and a part in affiliated non-university teaching hospitals.

\section{Questionnaire}

A validated Dutch translation of the original SCS was made. This was done by first having the survey translated from English into Dutch by a professional translator. A second professional translator translated the Dutch version back into English. No differences in meaning were found between the original version and the translated version. Respondents were asked to rate each item on a five-point Likert scale (disagree strongly, disagree slightly, neutral, agree slightly, agree strongly) or as not applicable. Table 1 outlines the 21 items of the SCS.

Table 1. Safety Climate Survey. Items, mean per item, percentage of respondents that scored the item $\geq 4.00$ and significant differences between females and males and university and affiliated non-university hospitals 
Martowirono, K., Wagner, C., Bijnen, A.B. Surgical residents' perceptions of patient safety climate in Dutch teaching hospitals. Journal of Evaluation in Clinical Practice: 2014, 20(2), 121128

\section{Distribution of the questionnaire}

The survey was administered as a distinctive section of a larger annual online survey among all surgical residents in the Netherlands which was commissioned by the Association of Surgical Residents in the Netherlands. It examines, amongst other things, surgical residents' demographics, perspectives on educational climate and workload, and vision of the future. The results were discussed with the Association of Surgeons in the Netherlands, as this association is responsible for surgical training in the Netherlands and attaches great importance to the opinions of the residents. The survey yielded high response rates in the past.

\section{Data management}

The SCS data and relevant respondent characteristics, such as gender, age, year of training, hospital and region of training, were provided in an Excel file by the researchers who held the large survey among residents. The data were entered into an SPSS file for analysis. Individual item responses were transposed into numerical values ranging from 1 to $5(1=$ disagree strongly, $2=$ disagree slightly, $3=$ neutral, $4=$ agree slightly, $5=$ agree strongly). Items that were answered as 'not applicable' were used for assessing face validity, but they were excluded from other calculations. Most items of the SCS are worded in such a way that higher ratings represent a more positive safety climate. Only item 20 (Personnel frequently disregard rules or guidelines that are established for this clinical area) is worded to reflect a negative element of safety climate. Therefore, the scoring of this question was scaled in reverse. For analysis, the hospitals were recoded into two categories $(1=$ university hospital, 2 = affiliated non-university hospital).

\section{Data analysis}

Potential usefulness of the SCS for assessing surgical residents' perceptions of the safety climate in Dutch teaching hospitals

Two psychometric properties were assessed to evaluate whether the SCS is a potentially useful instrument for measuring surgical residents' perceptions of the safety climate in Dutch teaching hospitals: face validity and internal consistency. Face validity assesses whether a test 'looks valid' and is assessed by intuitive judgement, rather than by rigorous statistical tests. In this study, face validity was assessed in two ways:

by asking the members of the Patient Safety Committee of the Association of Surgeons in the Netherlands to give their opinions on the relevance of the items of the SCS for measuring the perceptions of surgical residents regarding the patient safety climate in Dutch teaching hospitals; and

by evaluating how many times an item was rated as 'not applicable' by the surgical residents who participated in this study.

Internal consistency was established by assessing Cronbach's alpha. Cronbach's alpha examines whether several items that propose to measure the same construct produce similar scores. Ranges from 0.7 to 0.9 or up are considered to be acceptable [32]. In our study we chose a cut-off point of 0.7 . Furthermore, we examined whether deleting an item would change Cronbach's alpha. This information can be used to optimize the survey, keeping the adequate questions while replacing the inadequate questions. 
Martowirono, K., Wagner, C., Bijnen, A.B. Surgical residents' perceptions of patient safety climate in Dutch teaching hospitals. Journal of Evaluation in Clinical Practice: 2014, 20(2), 121128

\section{Surgical residents' perceptions of the patient safety climate in Dutch teaching} hospitals

We computed the overall SCS mean and the mean per individual SCS item. Because an ordinal five-point Likert scale was used to score the items of the SCS, we used the non-parametric Mann-Whitney test to determine if significant differences existed among two independent groups (gender, type of hospital) and the Kruskal-Wallis test to determine if significant differences existed among three or more independent groups (year of training, hospital, region of training). A cut-off score of 4.00 (agree slightly) was used to identify the items that the surgical residents valued negatively $(<4.00)$ or positively $(\geq 4.00)$. Furthermore, we ranked the means per individual SCS item in order to easily assess special areas of focus.

All statistical analyses were performed using IBM SPSS Statistics 20 for Windows (IBM Corp., Armonk, NY, USA) and PASW Statistics (formerly SPSS Statistics) 18 for Mac (SPSS Inc., Chicago, IL, USA). Statistical significance was defined as $\mathrm{P}<0.05$, two-sided.

\section{RESULTS}

\section{Respondents}

All surgical residents must be members of the Association of Surgical Residents in the Netherlands. At the time of the study, 390 surgical residents were registered at the association, all of whom received the large survey to which the SCS was attached. The large survey was completed by 315 surgical residents, 306 of whom completed the SCS. This resulted in an overall response rate of $78 \%(306 / 390)$. The mean age of the respondents was 31.71 years [standard deviation (SD) 2.56], with a minimum of 26 years and a maximum of 38 years. Table 2 shows the other respondent characteristics.

\section{POTENTIAL USEFULNESS OF THE SCS TO ASSESS SURGICAL RESIDENTS' PERCEPTIONS OF THE SAFETY CLIMATE IN DUTCH TEACHING HOSPITALS}

\section{Face validity}

The Patient Safety Committee of the Association of Surgeons in the Netherlands concluded that the items of the SCS were relevant for measuring the perceptions of surgical residents regarding the patient safety climate in Dutch teaching hospitals. The highest frequency of 'not applicable' was only $10 \%$ (item 6 - my suggestions about safety would be acted upon if I expressed them to management).

\section{Internal consistency}

The Cronbach's alpha found in this study was 0.87 . Deleting items had only a marginally positive effect on Cronbach's alpha or none at all. The highest, but still limited raise in Cronbach's alpha was achieved by deleting item 20 (personnel frequently disregards rules or guidelines that are established for this clinical area), the only negatively formulated item in the questionnaire (0.88). 
Martowirono, K., Wagner, C., Bijnen, A.B. Surgical residents' perceptions of patient safety climate in Dutch teaching hospitals. Journal of Evaluation in Clinical Practice: 2014, 20(2), 121128

\section{SURGICAL RESIDENTS' PERCEPTIONS OF THE PATIENT SAFETY CLIMATE IN DUTCH TEACHING HOSPITALS}

\section{Overall SCS mean and mean per individual SCS item}

Out of a maximum score of 5.00, the surgical residents reported an overall mean of 3.95 (SD 0.51), with a minimum of 2.11 and a maximum of 5.00. Of the respondents, $52 \%$ reported an overall mean of 4.00 or higher. Table 1 shows the mean per individual SCS item and the percentage of respondents that scored the item with 4.00 or higher.

\section{Differences between subgroups of surgical residents with respect to overall mean and mean per individual SCS item}

Table 2 shows the overall means reported by different subgroups of respondents. Figure 1 shows the overall means that were given by the surgical residents from the 55 different hospitals. The non-parametric tests showed that there were significant differences in overall SCS mean between men and women $(\mathrm{P}=0.00)$, among surgical residents from different regions $(\mathrm{P}=0.04)$ and between surgical residents from university hospitals and affiliated non-university hospitals $(\mathrm{P}=0.00)$. Women gave significantly lower scores than men, and residents from university hospitals gave significantly lower scores than residents from affiliated non-university hospitals. Further analyses showed that the differences in overall SCS means between university hospitals and affiliated non-university hospitals persisted when $25 \%$ of the lowest-scoring hospitals in both categories ( 2 university hospitals and 12 affiliated non-university hospitals) were excluded from the analyses. This showed that the difference was not determined by the two relatively low-scoring university hospitals (Fig. 1, hospitals 34 and 49). Significant differences in overall SCS mean were also found among surgical residents from different hospitals $(\mathrm{P}=0.009)$. Regarding the mean per individual SCS item, the non-parametric tests showed that most differences were found between men and women (11 items) and between surgical residents from university hospitals and affiliated non-university hospitals (8 items), with women giving significantly lower scores than men and with residents from university hospitals giving significantly lower scores than residents from affiliated nonuniversity hospitals (Table 1).

\section{[FIGURE 1.]}

\section{DISCUSSION}

\section{Potential usefulness of the SCS in assessing surgical residents' perceptions of the safety climate in Dutch teaching hospitals}

Regarding face validity, our findings suggest that the items of the SCS are relevant for measuring surgical residents' perceptions of the safety climate in Dutch teaching hospitals. Furthermore, the internal consistency of the questionnaire proved to be high, which is in line with the results of other studies [28]. Deleting items did not or only marginally improve the internal consistency. We therefore suggest maintaining all the items of the questionnaire when it is administered to Dutch surgical residents. Based on the assessment of face validity and internal consistency, we conclude that 
Martowirono, K., Wagner, C., Bijnen, A.B. Surgical residents' perceptions of patient safety climate in Dutch teaching hospitals. Journal of Evaluation in Clinical Practice: 2014, 20(2), 121128

the SCS is a potentially useful instrument for measuring perceptions of surgical residents regarding the patient safety climate in Dutch teaching hospitals.

\section{Surgical residents' perceptions of the patient safety climate in Dutch teaching hospitals}

In our study, we found an overall SCS mean of 3.95 (SD 0.51), with a minimum of 2.11 and a maximum of 5.00. In 2004, Kho et al. administered the SCS in four Canadian university-affiliated ICUs to all staff including nurses, allied health care professionals, non-clinical staff, intensivists and managers. They established a mean score of 3.5 for all participants (SD 0.6). Specifically for physicians, they found a mean score of 3.6 (SD 0.6) [30]. In 2007, Kho et al. administered a slightly modified version of the SCS to clinicians and non-clinicians of a Canadian medical-surgical ICU. They found a mean score of 4.0 (SD 0.6) and concluded that the safety climate score was encouraging, but that there still was room for improvement [33]. Our findings are similar to the findings of Kho et al. With an overall mean of 3.95 (SD 0.51 ), the surgical residents perceived the safety climate between 'neutral' and 'agree slightly'. Based on this finding and on the fact that only $52 \%$ of the respondents reported a positive climate (overall mean of 4.00 or higher), we conclude that there still is considerable room for improvement.

Women gave significantly lower scores than men. Apparently, in the Dutch setting, women are more critical than men in this respect. These differences were also found for several individual SCS items. Kho et al. found that in univariate analysis, male sex was associated with higher standardized safety climate scores, but no independent predictors of safety climate scores were found when multivariate multiple regression analysis was used [35]. Carney et al. studied sex differences in patient safety perceptions of operating room (OR) care givers, using the Safety Attitudes Questionnaire (OR version) [21,34]. This questionnaire measures care providers' perceptions of patient safety culture across six domains: teamwork climate, safety climate, job satisfaction, stress recognition, perceptions of management and working conditions. They found differences in mean scores between men and women for the following domains: teamwork, job satisfaction, management and working conditions, with women giving lower scores than men. No sex differences were found, however, for the domains safety climate and stress recognition. This difference between the study of Carney and our study might be explained by the differences between the questionnaires. The Safety Attitudes Questionnaire uses only seven items to measure safety climate. The SCS, however, consists of 21 items, including six of the seven safety climate items of the Safety Attitudes Questionnaire. Furthermore, our study focused on relatively young surgical residents who are still in training, whereas Carney included surgeons who had many more years in the profession. Reasons for the gender difference found in various studies, including ours, are speculative. It might be explained by the fact that while female surgical residents constitute quite an influential mass in our country (37\%), their supervisors are still predominantly male [35]. Women might be more safetyoriented than men and more likely to communicate this in these male surroundings. Surgical residents who work in university hospitals reported lower scores than residents who work in affiliated non-university hospitals. These findings are partially comparable to the findings of Carney et al., who reported differences in perceptions of safety climate between high-complexity and medium-complexity facilities [36, 
37]. We do not know the reason for the difference found between these two types of hospitals, but it might be that in the more complex organization of university hospitals, workers are listened to less readily and actions are carried out more slowly. We also found a large variability in the overall means of the perceptions of residents from different hospitals (range 3.20-4.60). In the future, it might be interesting to repeat this survey and provide each hospital with their data as well as with a set of reference data, for example the overall means per item that were found during the survey. This would enable the hospitals to compare themselves to these reference data. The combination of data could elicit specific points of improvement that a hospital should focus on.

Table 1 shows that surgical residents perceived more than half of the items negatively. The variety of negatively valued items indicates many opportunities for improvement. The residents reported relatively positive perceptions regarding briefings (items 12 and 13). Developing, implementing and evaluating briefings have been important parts of patient safety improvement efforts. It seems that these briefings have found their way into practice.

\section{Opportunities for safety climate improvement through changes in surgical training in the Netherlands}

Regarding surgical training in the Netherlands, several items deserve special attention. The residents reported less favourable perceptions regarding feedback (item 1). Furthermore, the residents reported that the culture of the clinical area does not make it easy to learn from mistakes made by others (item 10). In other words, residents do not perceive sufficient opportunity to learn from their own performance or from the performance of others. Based on these findings, we recommend reviewing and optimizing the existing feedback processes and incorporating explicit feedback on issues related to patient safety. To improve the opportunities for learning from the mistakes of others, we recommend focusing periodically on patient safety during formal teaching sessions. On condition that confidentiality is guaranteed, these sessions provide the opportunity to discuss adverse events and the factors that caused them. The sessions can also be used for so-called executive walk rounds [38], that is visits by hospital executives to patient care areas to discuss patient safety issues with providers. This may have a positive impact on the perception of the reinforcement of patient safety as priority (item 21), the perception that senior leaders listen to and care about surgical residents' concerns (item 3 ) and the perception that suggestions about safety would be acted upon (item 6).

During residency, surgical residents work in several hospitals. Table 1 shows that surgical residents do not know the proper channels for addressing questions regarding patient safety (item 9). We think that this issue can easily be solved, for instance by paying attention to it during introduction courses for new residents. Furthermore, supervisors should encourage surgical residents to report any patient safety concern that they may have.

\section{STRENGTHS}

The strength of this study is the high response rate, realized by taking part in a large online survey among surgical residents that yielded high response rates in the past. It reflects the opinions of the surgical residents, which is of key importance for surgical 
Martowirono, K., Wagner, C., Bijnen, A.B. Surgical residents' perceptions of patient safety climate in Dutch teaching hospitals. Journal of Evaluation in Clinical Practice: 2014, 20(2), 121128

training programs. It describes an instrument that can easily be used for follow-up research on perceived safety climate. The study offers immediately practicable points for improvement.

\section{LIMITATIONS}

Kho et al. conclude in their paper on the three different instruments to measure patient safety (SCS, Safety Culture Scale and Safety Climate Mean) that the SCS appears to measure one construct and is sufficiently reliable [30]. Pronovost and Sexton appreciate the work done by Kho et al., but they emphasize that the items of the SCS are a subset, related to safety climate, from the larger Safety Attitudes Questionnaire. This subset of safety climate items does not elicit attitudes along any dimensions such as teamwork climate or perceptions of management. Pronovost and Sexton state that it might be more valuable to use multidimensional cultural assessments [29]. The Safety Attitudes Questionnaire consists of 58 items. It shows good psychometric properties and is often used to measure and compare caregiver attitudes about six patient safety-related domains [21]. We acknowledge the value of using multidimensional cultural assessments. In view of a high response and because the number of items that we could attach to the larger survey was limited, we decided, however, to use the shorter SCS questionnaire.

We attempted to take a first step in evaluating the SCS for use among surgical residents in the Netherlands by assessing face validity and internal consistency. Further research on other aspects of reliability and validity is required in order to get a complete overview of the appropriateness of using the SCS for measuring the perceptions of surgical residents regarding the patient safety climate in Dutch teaching hospitals.

Furthermore, we only asked surgical residents to participate in our study. The surgical resident is a member of a team that also includes supervisors, nurses and other professionals, including other medical specialists. In order to get more detailed and complete information on the prevailing safety climate, it might be interesting to include those other team members in future surveys. However, performing such larger surveys has its own disadvantages, such as lower practicability, presumably lower response rates and higher costs. As the opinions of the surgical residents themselves are paramount, we feel that setting such a simple, cheap and easily repeatable questionnaire is a justified approach (Box 1).

\section{[Box 1]}

\section{CONCLUSIONS}

Based on the assessment of face validity and internal consistency, we conclude that the SCS is a potentially useful instrument to measure perceptions of surgical residents regarding the patient safety climate in Dutch teaching hospitals. The perceptions of surgical residents on safety climate can be improved considerably, as only $52 \%$ of the respondents reported a positive climate (overall mean of 4.00 or higher on a five-point Likert scale). Regarding surgical training, feedback processes should be reviewed and optimized. Furthermore, formal teaching sessions with a focus on patient safety should be periodically organized at the workplace, attention should be paid to channels for safety-related questions during the introduction in a 
Martowirono, K., Wagner, C., Bijnen, A.B. Surgical residents' perceptions of patient safety climate in Dutch teaching hospitals. Journal of Evaluation in Clinical Practice: 2014, 20(2), 121128

new hospital, and supervisors should encourage surgical residents to report any patient safety concern they may have.

\section{ACKNOWLEDGEMENT}

We would like to thank Tjeerd van der Ploeg, statistician, for his assistance in analysing the data using SPSS.

This study was supported in part by the Stichting Kwaliteitsgelden Medisch Specialisten in the Netherlands (SKMS, Quality foundation of the Dutch Medical Specialists).

\section{REFERENCES}

1 Kohn, L. T., Corrigan, J. \& Donaldson, M. S. (1999) To Err Is Human: Building A Safer Health System. Washington, D.C.: National Academy Press.

2 Zegers, M., De Bruijne, M. C., Wagner, C., et al. (2009) Adverse events and potentially preventable deaths in Dutch hospitals: results of a retrospective patient record review study. Quality and Safety in Health Care, 18 (4), 297-302.

3 De Vries, E. N., Ramrattan, M. A., Smorenburg, S. M., Gouma, D. J. \& Boermeester, M. A. (2008) The incidence and nature of in-hospital adverse events: a systematic review. Quality and Safety in Health Care, 17 (3), 216-223.

4 Nundy, S., Mukherjee, A., Sexton, J. B., Ponovost, P. J., Knight, A., Rowen, L. C., Duncan, M., Syin, D. \& Makary, M. A. (2008) Impact of preoperative briefings on operating room delays: a preliminary report. Archives of Surgery, 143 (11), 1068-1072.

5 Haynes, A. B., Weiser, T. G., Berry, W. R., et al. (2009) A surgical safety checklist to reduce morbidity and mortality in a global population. The New England Journal of Medicine, 360 (5), 491-499.

6 Semel, M. E., Resch, S., Haynes, A. B., Funk, L. M., Bader, A., Berry, W. R., Weiser, T. G. \& Gawande, A. A. (2010) Adopting a surgical safety checklist could save money and improve the quality of care in U.S. hospitals. Health Affairs, 29 (9), 1593-1599.

7 Einav, Y., Gopher, D., Kara, I., Ben-Yosef, O., Lawn, M., Laufer, N., Liebergall, M. \& Donchin, Y. (2010) Preoperative briefing in the operating room: shared cognition, teamwork, and patient safety. Chest, 137 (2), 443-449.

8 De Vries, E. N., Prins, H. A., Crolla, R. M. P. H., et al. (2010) Effect of a comprehensive surgical safety system on patient outcomes. The New England Journal of Medicine, 363 (20), 1928-1937.

9 Frank, J. R. \& Brien, S. (eds) The Safety Competencies Steering Committee (2008) The Safety Competencies: Enhancing Patient Safety Across the Health Professions. Ottawa, ON: Canadian Patient Safety Institute.

10 Alonso, A., Baker, D. P., Holtzman, A., Day, R., King, H., Toorney, L. \& Salas, E. (2006) Reducing medical error in the military health system: how can team training help? Human Resource Management Review, 16, 396-415

11 Aron, D. C. \& Headrick, L. A. (2002) Educating physicians prepared to improve care and safety is no accident: it requires a systematic approach. Quality and Safety in Health Care, $11,168-173$.

12 Lucian Leape Institute at the National Patient Safety Foundation (2010) Unmet needs. Teaching physicians to provide safe patient care. Available at: http://www.npsf.org/wpcontent/uploads/2011/10/LLI-Unmet-Needs-Report.pdf (accessed 28 June 2012).

13 Jansma, J. D., Wagner, C. \& Bijnen, A. B. (2011) A patient safety curriculum for medical residents based on the perspectives of residents and supervisors. Journal of Patient Safety, 7, 99-105.

14 Jansma, J. D., Wagner, C. \& Bijnen, A. B. (2010) Residents' intentions and actions after patient safety education. BMC Health Services Research, 10, 350.

15 Jansma, J. D., Zwart, D. L. M., Leistikow, I. P., Kalkman, C. J., Wagner, C. \& Bijnen, A. B. (2010) Do specialty registrars change their attitudes, intentions and behaviour towards 
Martowirono, K., Wagner, C., Bijnen, A.B. Surgical residents' perceptions of patient safety climate in Dutch teaching hospitals. Journal of Evaluation in Clinical Practice: 2014, 20(2), 121128

reporting incidents following a patient safety course. BMC Health Services Research, 10, 100.

16 Jansma, J. D., Wagner, C., Ten Kate, R. W. \& Bijnen, A. B. (2011) Effects on incident reporting after educating residents in patient safety: a controlled study. BMC Health Services Research, 11, 335.

17 Reason, J. (1995) Understanding adverse events: human factors. Quality in Health Care, 4, 80-89.

18 Vincent, C., Taylor-Adams, S. \& Stanhope, N. (1998) Framework for analysing risk and safety in clinical medicine. British Medical Journal, 316, 1154-1157.

19 INSAG (International Nuclear Safety Advisory Group) (1991) Safety culture. 75-INSAG-4: $1-44$.

20 Flin, R., Burns, C., Mearns, K., Yule, S. \& Robertson, E. M. (2006) Measuring safety climate in health care. Quality and Safety in Health Care, 15, 109-115.

21 Sexton, J. B., Helmreich, R. L., Neilands, T. B., Rowan, K., Vella, K., Boyden, J., Roberts, P. R. \& Thomas, E. J. (2006) The Safety Attitudes Questionnaire: psychometric properties, benchmarking data, and emerging research. BMC Health Services Research, 6, 44.

22 Singer, S., Lin, S., Falwell, A., Gaba, D. \& Baker, L. (2009) Relationship of safety climate and safety performance in hospitals. Health Services Research, 44, 399-421.

23 Huang, D. T., Clermont, G., Kong, L., Weissfeld, L. A., Sexton, J. B., Rowan, K. M. \& Angus, D. C. (2010) Intensive care unit safety culture and outcomes: a US multicenter study. International Journal for Quality in Health Care, 22 (3), 151-161.

24 Rosen, A. K., Singer, S., Zhao, S., Shokeen, P., Meterko, M. \& Gaba, D. (2010) Hospital safety climate and safety outcomes: is there a relationship in the VA. Medical Care Research and Review, 67 (5), 590-608.

25 Mardon, R. E., Khanna, K., Sorra, J., Dyer, N. \& Famolaro, T. (2010) Exploring relationships between hospital patient safety culture and adverse events. Journal of Patient Safety, 6, 226-232.

26 Sexton, J. B. \& Thomas, E. J. (2003) The Safety Climate Survey: Psychometric and Benchmarking Properties. Technical Report 03-03. Austin, TX: The University of Texas Center of Excellence for Patient Safety Research and Practice. (AHRQ grant \# 1PO1HS1154401 and U18HS1116401).

27 Singla, A. K., Kitch, B. T., Weissman, J. S. \& Campbell, E. G. (2006) Assessing patient safety culture: a review and synthesis of the measurement tools. Journal of Patient Safety, $2,105-115$.

28 Colla, J. B., Bracken, A. C., Kinney, L. M. \& Weeks, W. B. (2005) Measuring patient safety climate: a review of surveys. Quality and Safety in Health Care, 14, 364-366.

29 Pronovost, P. \& Sexton, B. (2005) Assessing safety culture: guidelines and recommendations. Quality and Safety in Health Care, 14, 231-233.

30Kho, M. E., Carbone, J. M., Lucas, J. \& Cook, D. J. (2005) Safety Climate Survey: reliability of results from a multicenter ICU survey. Quality and Safety in Health Care, 14, 273-278.

31 Etchegaray, J. M. \& Fischer, W. (2006) Survey research: be careful where you step .... Quality and Safety in Health Care, 15, 154-155.

32 Cronbach, L. J. (1951) Coefficient alpha and the internal structure of tests. Psychometrika, 16 (3), 297-334.

33 Kho, M. E., Perri, D., McDonald, E., Waugh, L., Orlicki, C., Monaghan, E. \& Cook, D. J. (2009) The climate of patient safety in a Canadian intensive care unit. Journal of Critical Care, 24, 469e7-469e13.

34 Carney, B. T., Mills, P. D., Bagian, J. P. \& Weeks, W. B. (2010) Sex differences in operating room care giver perceptions of patient safety: a pilot study from the Veterans Health Administration Medical Team Training Program. Quality and Safety in Health Care, 19, 128-131.

35 Kanter, R. M. (1977) Men and Women of the Corporation. New York: Basic Books.

36 Carney, B. T., West, P., Neily, J., Mills, P. D. \& Bagian, J. P. (2010) The effect of facility complexity on perceptions of safety climate in the operating room: size matters. American Journal of Medical Quality, 25 (6), 457-461. 
Martowirono, K., Wagner, C., Bijnen, A.B. Surgical residents' perceptions of patient safety climate in Dutch teaching hospitals. Journal of Evaluation in Clinical Practice: 2014, 20(2), 121128

37 Carney, B. T., West, P., Neily, J., Mills, P. D. \& Bagian, J. P. (2011) Changing perceptions of safety climate in the operating room with the Veterans Health Administration Medical Team Training Program. American Journal of Medical Quality, 26 (3), 181-184.

38 Thomas, E. J., Sexton, J. B., Neilands, T. B., Frankel, A. \& Helmreich, R. L. (2005) The effect of executive walkrounds on nurse safety climate attitudes: a randomized trial of clinical units. BMC Health Services Research, 5, 28.

\section{TABLES, FIGURES AND BOX}

Table 1. Safety Climate Survey. Items, mean per item, percentage of respondents that scored the item $\geq 4.00$ and significant differences between females and males and university and affiliated non-university hospitals

\begin{tabular}{|c|c|c|c|c|c|c|c|c|c|c|c|}
\hline \multirow{2}{*}{$\begin{array}{l}\text { Item } \\
\text { no. }\end{array}$} & \multirow[b]{2}{*}{ Item } & \multirow[b]{2}{*}{$N^{x}$} & \multirow[b]{2}{*}{ Mean } & \multirow[b]{2}{*}{ SD } & \multirow{2}{*}{$\geq 4.00$} & \multicolumn{2}{|c|}{ Gender } & \multirow[b]{2}{*}{$P$} & \multicolumn{2}{|c|}{ Type of hospital } & \multirow[b]{2}{*}{$P$} \\
\hline & & & & & & $\mathrm{F}$ & M & & $\mathrm{U}$ & ANU & \\
\hline 12 & $\begin{array}{l}\text { Briefing personnel before the start of a shift li.e., to } \\
\text { plan for possible contingencias) is an important part } \\
\text { of patient safety. }\end{array}$ & 303 & 4.62 & 0.68 & 92.8 & & & & & & \\
\hline 13 & Briefings are common here. & 303 & 4.47 & 0.85 & 89.1 & & & & & & \\
\hline 14 & $\begin{array}{l}\text { I am satisfied with availability of clinical leadership: } \\
\text { physician }\end{array}$ & 303 & 4.31 & 0.90 & 86.5 & & & & & & \\
\hline 2 & $\begin{array}{l}\text { Medical errors are handled appropriately in this clinical } \\
\text { area. }\end{array}$ & 302 & 4.12 & 0.91 & 80.5 & 3.88 & 4.26 & 0.001 & 3.86 & 4.25 & 0.001 \\
\hline 4 & $\begin{array}{l}\text { The physician and nurse leaders in my area listen to } \\
\text { me and care about my concerns. }\end{array}$ & 298 & 4.12 & 0.93 & 79.5 & 3.91 & 4.24 & 0.003 & 3.91 & 4.23 & 0.005 \\
\hline 5 & $\begin{array}{l}\text { Leadership is driving us to be a safety-centred } \\
\text { institution. }\end{array}$ & 304 & 4.12 & 0.97 & 78.0 & & & & & & \\
\hline 11 & I would feel safe baing treated here as a patient. & 305 & 4.05 & 0.98 & 78.3 & 3.90 & 4.14 & 0.029 & 3.67 & 4.25 & 0.000 \\
\hline 7 & $\begin{array}{l}\text { Management/eadership does not knowingly } \\
\text { compromise safety concerns for productivity. }\end{array}$ & 290 & 4.04 & 1.08 & 72.7 & & & & & & \\
\hline 18 & $\begin{array}{l}\text { I believe that most adverse events occur as a result of } \\
\text { multiple system failures, and are not attributable to } \\
\text { one individual's actions. }\end{array}$ & 303 & 4.02 & 0.96 & 75.9 & 3.87 & 4.10 & 0.039 & & & \\
\hline 19 & $\begin{array}{l}\text { The personnel in this clinical area take responsibility for } \\
\text { patient safety. }\end{array}$ & 300 & 3.99 & 0.84 & 77.3 & & & & & & \\
\hline 1 & $\begin{array}{l}\text { The culture of this clinical area makes it easy to learn } \\
\text { from the mistakes of others. }\end{array}$ & 305 & 3.94 & 1.04 & 74.8 & 3.73 & 4,06 & 0.012 & 3.59 & 4.12 & 0.000 \\
\hline 17 & $\begin{array}{l}\text { This institution is doing more for patient safety now } \\
\text { than it did one year ago. }\end{array}$ & 275 & 3.89 & 0.88 & 62.1 & & & & & & \\
\hline 16 & $\begin{array}{l}\text { I am satisfied with availability of clinical leadership: } \\
\text { pharmacy }\end{array}$ & 290 & 3.86 & 0.94 & 64.1 & & & & & & \\
\hline 20 & $\begin{array}{l}\text { Personnel frequently disregards rules or guidelines that } \\
\text { are established for this clinical area (reverse). }\end{array}$ & 297 & 3.82 & 0.98 & 72.7 & & & & & & \\
\hline 10 & I receive appropriate feedback about my performance. & 304 & 3.79 & 1.00 & 71.0 & 3.62 & 3.90 & 0.032 & 3.59 & 3.90 & 0.010 \\
\hline 9 & $\begin{array}{l}\text { I know the proper channels to direct questions } \\
\text { regarding patient safety. }\end{array}$ & 302 & 3.72 & 1.09 & 67.2 & 3.43 & 3.89 & 0.000 & & & \\
\hline 15 & $\begin{array}{l}\text { I am satisfied with availability of clinical leadership: } \\
\text { nursing }\end{array}$ & 298 & 3.70 & 1.09 & 64.7 & 3,44 & 3.85 & 0.001 & 3,49 & 3.81 & 0.047 \\
\hline 3 & $\begin{array}{l}\text { The senior leaders in my hospital listen to me and care } \\
\text { about my concerns. }\end{array}$ & 290 & 3.69 & 1.09 & 60.3 & 3,46 & 3.82 & 0.010 & 3.47 & 3.81 & 0.018 \\
\hline 21 & $\begin{array}{l}\text { Patient safety is constantly reinforced as the priority in } \\
\text { this clinical area. }\end{array}$ & 300 & 3.69 & 0.95 & 62.0 & & & & & & \\
\hline 6 & $\begin{array}{l}\text { My suggestions about safety would be acted upon if I } \\
\text { expressed them to management. }\end{array}$ & 274 & 3.56 & 1.04 & 57.3 & 3.28 & 3.74 & 0.001 & 3.35 & 3.68 & 0.016 \\
\hline 8 & $\begin{array}{l}\text { I am encouraged by my colleagues to report any patient } \\
\text { safety concerns I may have. }\end{array}$ & 297 & 3.41 & 1.05 & 48.5 & 3.16 & 3.56 & 0.001 & & & \\
\hline
\end{tabular}

'Items that were answered as 'not applicable' were excluded from the analyses. Items below the bold line scored a mean less than 4.00 . Grey areas indicate that no significant differences are found.

ANU, affiliated non-university hospital; SD, standard deviation; U, university hospital.

1 = disagree strongly, 2 = disagree slightly, $3=$ neutral, 4 = agree slightly, 5 = agree strongly. 
Martowirono, K., Wagner, C., Bijnen, A.B. Surgical residents' perceptions of patient safety climate in Dutch teaching hospitals. Journal of Evaluation in Clinical Practice: 2014, 20(2), 121128

Table 2.: Respondent characteristics. Overall mean for subgroups of respondents and $P$ value for differences in overall mean between subgroups of respondents

\begin{tabular}{|c|c|c|c|c|c|c|}
\hline \multicolumn{2}{|c|}{ Respondent characteristics } & \multirow{2}{*}{\begin{tabular}{|l}
$\mid n$ \\
113 \\
\end{tabular}} & \multirow{2}{*}{\begin{tabular}{|l|}
$\%$ \\
36.9 \\
\end{tabular}} & \multirow{2}{*}{\begin{tabular}{|l|}
$\begin{array}{l}\text { Overall } \\
\text { mean }\end{array}$ \\
3.82 \\
\end{tabular}} & \multirow{2}{*}{\begin{tabular}{|l|} 
SD \\
0.49
\end{tabular}} & \multirow{3}{*}{$\mid \begin{array}{ll}\mid \boldsymbol{P} \text { value } \\
0.000\end{array}$} \\
\hline \multirow{2}{*}{ Gender } & Female & & & & & \\
\hline & Male & 193 & 63.1 & 4.03 & 0.51 & \\
\hline \multirow{6}{*}{ Year of training } & Year 1 & 40 & 13.1 & 4.02 & 0.47 & \multirow{6}{*}{0.801} \\
\hline & Year 2 & 49 & 16.0 & 3.94 & 0.47 & \\
\hline & Year 3 & 40 & 13.1 & 3.86 & 0.54 & \\
\hline & Year 4 & 46 & 15.0 & 3.96 & 0.55 & \\
\hline & Year 5 & 59 & 19.3 & 3.97 & 0.48 & \\
\hline & Year 6 & 72 & 23.5 & 3.95 & 0.55 & \\
\hline \multirow{8}{*}{$\begin{array}{l}\text { Region of } \\
\text { training }\end{array}$} & A & 23 & 7.5 & 3.74 & 0.68 & \multirow{8}{*}{0.041} \\
\hline & $\mathrm{B}$ & 40 & 13.1 & 4.06 & 0.53 & \\
\hline & $\mathrm{C}$ & 23 & 7.5 & 4.05 & 0.43 & \\
\hline & $\mathrm{D}$ & 47 & 15.4 & 3.84 & 0.59 & \\
\hline & $\mathrm{E}$ & 35 & 11.4 & 3.89 & 0.41 & \\
\hline & $\mathrm{F}$ & 50 & 16.3 & 3.92 & 0.49 & \\
\hline & $\mathrm{G}$ & 45 & 14.7 & 4.12 & 0.47 & \\
\hline & $\mathrm{H}$ & 43 & 14.1 & 3.96 & 0.42 & \\
\hline \multirow[b]{2}{*}{ Type of hospital } & University & 202 & 66 & 4.01 & 0.50 & \multirow[b]{2}{*}{0.003} \\
\hline & $\begin{array}{l}\text { Affiliated non- } \\
\text { university }\end{array}$ & 104 & 34 & 3.83 & 0.52 & \\
\hline
\end{tabular}

1. SD, standard deviation. 
Martowirono, K., Wagner, C., Bijnen, A.B. Surgical residents' perceptions of patient safety climate in Dutch teaching hospitals. Journal of Evaluation in Clinical Practice: 2014, 20(2), 121128

Figure 1.: Overall mean per hospital. X-axis: hospital coded by number; grey, university hospitals; black, affiliated non-university hospitals. Y-axis: overall mean per hospital.

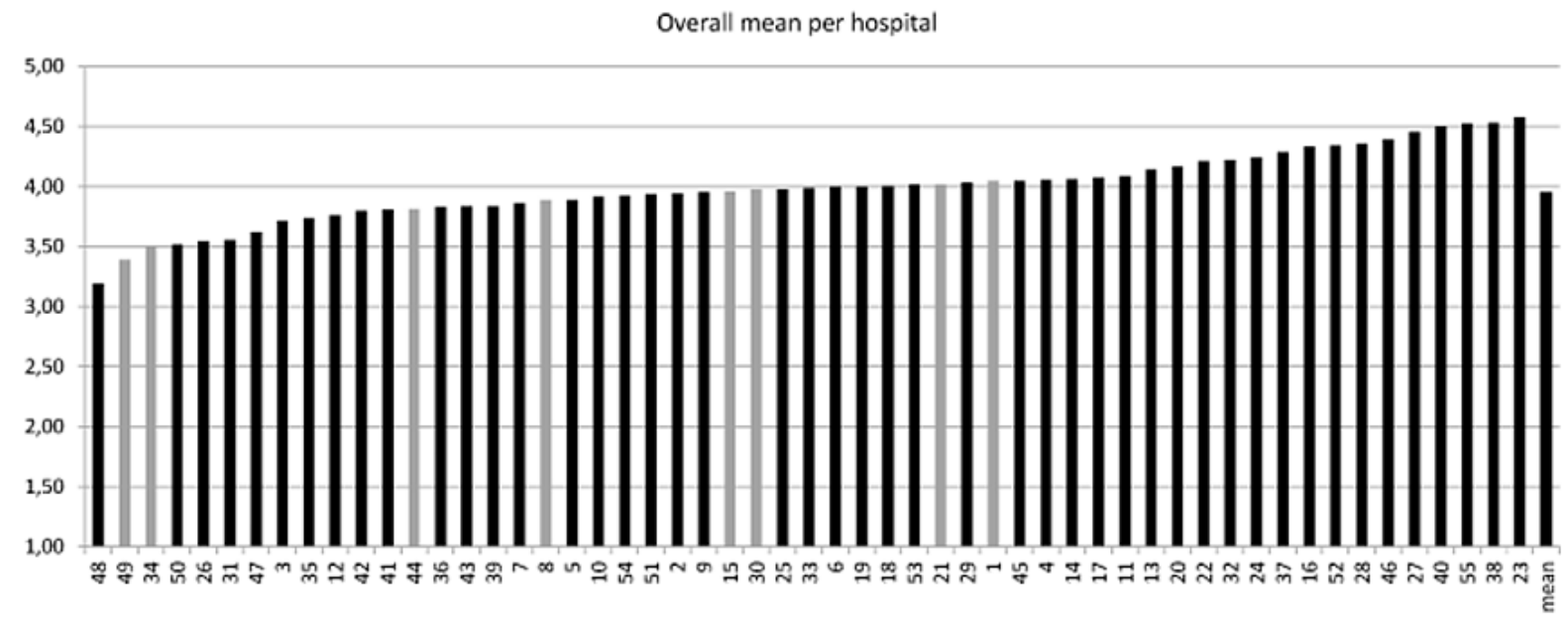

Box 1. Practical recommendations for the Association of Surgeons in the Netherlands

- $\quad$ Safety Climate Survey

o Administer the SCS periodically to surgical residents as a part of the larger survey among these residents. The results can be used to monitor (changes in) perceptions of safety climate and to highlight opportunities for safety climate improvement.

o Conduct further research on other aspects of validity and reliability.

o Consider to include other team members in future surveys.

- Surgical training in the Netherlands

o Review and optimize feedback processes.

o Include formal teaching sessions at the workplace with a focus on patient safety.

o Pay attention to patient safety during the introduction of surgical residents in new hospitals.

o Encourage surgical residents to report patient safety concerns. 